\title{
Mechanical plus pharmacological prophylaxis for deep vein thrombosis in acute spinal cord injury
}

\author{
G J Merli MD FACP, ${ }^{1}$ S Crabbe PharmD,${ }^{2}$ L Doyle BSN,${ }^{1}$ J F Ditunno MD FACP, ${ }^{3}$ \\ G J Herbision MD FACP ${ }^{3}$
}

${ }^{1}$ Department of Medicine, ${ }^{2}$ Department of Pharmacy, ${ }^{3}$ Department of Rehabilitation Medicine $21159^{\text {th }}$ Street, $3^{\text {rd }}$ Floor, Thomas Jefferson University Hospital, Philadelphia, Pa 19107, USA and the Regional Spinal Cord Injury Center of the Delaware Valley, Philadelphia, $\mathrm{Pa}$, USA.

This pilot study assessed the effectiveness of external pneumatic compression (EPC) with gradient elastic stockings (GES) and low dose heparin (LDH) in the prevention of deep vein thrombosis (DVT) in acute spinal cord injured (SCI) patients in the first 14 days following injury. Twenty-one consecutive patients within $48 \mathrm{hrs}$ of injury with acute C2 through T12 motor complete or nonfunctional motor incomplete spinal cord lesions were enrolled. 125 I fibrinogen scanning was performed daily. A positive scan was confirmed by venography. Thrombosis was demonstrated in $1 / 19$ patients. A control group from a previous study confirmed thrombosis in 6/17 patients $(p=0.04)$. Comparison of extent of thrombosis demonstrated a reduction in the treated group $(p=0.02)$. This pilot study demonstrates that EPC with GES plus LDH may be an effective prophylactic regimen in these patients.

Key words: deep vein thrombosis; spinal cord injury; prophylaxis; mechanical and pharmalogical.

\section{Introduction}

The number of new spinal cord injuries in the United States continues to increase each year. ${ }^{1}$ Deep vein thrombosis (DVT) and pulmonary embolism (PE) are the major post injury complications in the Frankel A, $\mathrm{B}$, and $\mathrm{C}$ groups with an incidence ranging from $49 \%$ to $72 \%$ (Table I). ${ }^{2-5}$ They will usually occur in the first 2 weeks following injury. ${ }^{5,6}$ Various modalities of prophylaxis adapted from the surgical literature such as subcutaneous heparin $(5,000 \mathrm{u}, \mathrm{SC}, \mathrm{Q} 8 \mathrm{hrs})$ and warfarin have been ineffective in reducing the incidence of DVT in this population. ${ }^{5-7}$ More recently, combination therapies which reduce stasis and hypercoagulability have achieved a successful reduction in the incidence of DVT and PE but have not gained widespread acceptance. ${ }^{5,8}$ Reasons for this may be the lack of knowledge of the defined risk of DVT/PE, difficulty in application of prophylactic devices, or placement of the patient into aggressive rehabilitation programs. The purpose of this pilot study is to assess the combination of external pneumatic compression (EPC) sleeves plus gradient elastic stockings (GES) and subcutaneous low dose heparin (LDH) as prophylaxis for DVT in the first 2 weeks following acute SCI.

\section{Methodology}

Patients were included in the study if they were older than age 15 and had incurred a C2 through T12 motor complete or motor

Table I Frankel classification of spinal cord injury

$\begin{array}{ll}\text { Frankel class } & \begin{array}{l}\text { Neurological description } \\ \text { Complete: motor \& sensory } \\ \text { absent }\end{array} \\ \text { B } & \begin{array}{l}\text { Incomplete: preserved sensory, } \\ \text { absent motor } \\ \text { Incomplete: preserved motor, } \\ \text { nonfunctional }\end{array} \\ \text { C } & \begin{array}{l}\text { Incomplete: preserved motor, } \\ \text { functional } \\ \text { Complete return of motor \& } \\ \text { sensory function }\end{array} \\ \text { E } & \end{array}$


nonfunctional acute spinal cord injury within the past 72 hours. Patients were excluded if they had an underlying bleeding disorder, recent ( $<6$ months) myocardial infarction, long bone fracture, arterial trauma, postphlebitic syndrome, lower extremity cellutitis, hepatic (aspartate aminotransferase, lactate dehydrogenase, alkaline phosphatase) or renal (serum creatinine) function values twice normal. Pregnant patients and patients receiving anticoagulant drugs were also excluded. Informed consent was obtained from all patients after the nature of the procedures were explained.

Patients received prophylaxis with EPC plus GES and LDH. EPC was accomplished using a knee length sequential compression device (Kendall, Boston, Mass). The GES were calf length (Carolan's Type 511). The combination of sleeves and stockings were worn 23 hours per day with one hour off for skin care and bathing. Heparin sodium (Wyeth-Ayerst Laboratories, Philadelphia, PA) was administered at a dose of 5,000 units subcuntaneously in the abdomen on a 12 hour schedule at $0800 \mathrm{hrs}$ and $2000 \mathrm{hrs}$ daily. If patients underwent surgery, the morning dose was given 2 hours prior to surgery. 125 I fibrinogen scanning using the method of $\mathrm{Kakkar}^{9}$ was initiated within 18 hours of admission and performed daily for 2 weeks. The criteria for diagnosis of DVT was an increased uptake of $20 \%$ or more on 2 consecutive measurements compared with adjacent readings. All positive studies were confirmed by venography. All venograms were completed via the Rabinov et al technique and scored by the modified Marder et al deep vein thrombosis scoring scale (Table II). ${ }^{0,11}$ All venograms were interpreted by a blinded observer.

The 21 consecutive patients corresponded closely with respect to age, sex, level of lesion, surgery and length of 125 I fibrinogen scanning to our previously reported control group (Fig 1). ${ }^{5}$ This control group of 17 patients was used for comparison to our treatment group. The Fischer's Exact Test with a two-tailed probability was used to compare the incidence of DVT between the treated and control groups. The Ordered Chi Square Test that considers trend with a two-tailed probability was utilized to compare the degree of thrombosis demonstrated in each of the 2 groups. ${ }^{12}$

\section{Results}

Twenty-one consecutive patients entered the protocol within 72 hours of acute spinal cord injury. There were 5 women and 16 men ranging in age from 18 to 70 years. The level of injury included 16 cervical and 5 thoracic lesions (Table III). Two patients were not included in the evaluation because they violated the 72 hour entry window for initiating prophylaxis with $\mathrm{EPC}+\mathrm{LDH}$ + GES. One patient had been delayed 96 hours before EPC sleeves were placed and the second did not receive $\mathrm{LDH}$ for 96

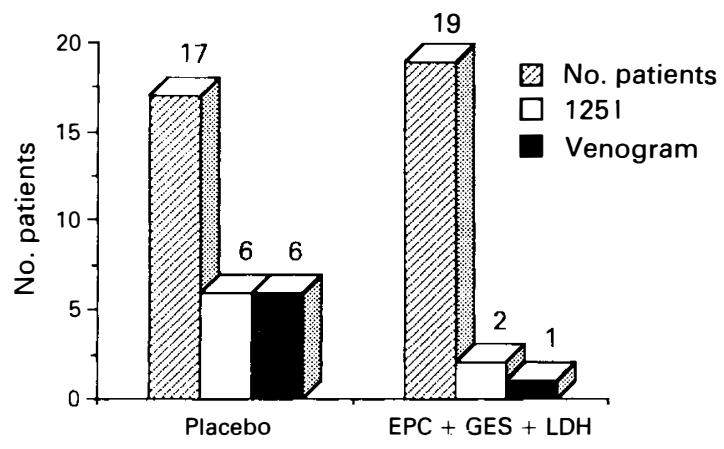

Figure 1 Deep vein thrombosis assessment

Table II Modified Marder Scoring Scale for DVT (adapted from Marder) ${ }^{11}$

\begin{tabular}{llcccc}
\hline Region & Occlusion & none & $<1 / 3$ & $1 / 3-2 / 3$ & $>2 / 3$ \\
\hline Pelvis & Illiac & 0 & 2 & 4 & 6 \\
Groin & Femoral & 0 & 1 & 2 & 4 \\
Thigh & Sup. femoral & 0 & 3 & 6 & 10 \\
Knee & Popliteal & 0 & 1 & 2 & 4 \\
Calf & Anterior tibial & 0 & 1 & 2 & 4 \\
& Posterior tibial & 0 & 2 & 4 & 6 \\
& Peroneal & 0 & 2 & 4 & 6 \\
\hline
\end{tabular}


Table III Characteristics of the 2 groups

\begin{tabular}{lcc}
\hline Characteristics & Control (17) & Treatment (19) \\
\hline Age range & $15-69$ & $18-70$ \\
Sex M:F & $11: 6$ & $14: 5$ \\
Frankel class & & \\
$\quad$ A & 15 & 17 \\
B & 2 & 2 \\
Level of lesion & & \\
$\quad$ Cervical & 13 & 14 \\
$\quad$ Thoracic & 4 & 5 \\
Surgery & 15 & 12 \\
\hline
\end{tabular}

hours. These patients did not remain part of the protocol and received the standard therapy of the center $(\mathrm{EPC}+\mathrm{LDH})$. One of the 2 developed a thrombosis 9 days post injury. Of the 19 evaluable patients, 17 $(89 \%)$ had negative fibrinogen scanning upon completion of the study. The 2 remaining patients $(11 \%)$ developed a positive fibrinogen scan on days 6 and 8 of the study (Fig 1). Venography was performed and demonstrated acute thrombosis in only one of these patients (5.2\%) (Fig 1). The thrombosis involved the tibialis anterior vein with a modified Marder Scale score of eight. In comparison, the control group developed positive 125 I fibrinogen scans in $6 / 17(35 \%)$ patients and all were confirmed by venography (Fig 1 ). The six thrombi found in the control group ranged from 8 to 40 on the Marder scoring scale with scores of $8,10,20,24,34,40$ (Fig 2). This incidence of thrombosis was significantly different than that of the treated group $(p=0.04)$. The extent of the clot was then compared between the 2 groups using a modified Marder score of $<10$ as minimal clot and $>10$ substantial clot. Using these assumptions a significant difference was found in the extent of clot between the 2 groups in favor of the EPC plus GES and LDH treated group $(p=0.02)$.

\section{Discussion}

Approaches to prophylaxis for DVT have varied from no treatment to single agent or combination modalities. ${ }^{13}$ These methods have attempted to improve one or more arms of Virchow's Triad which includes

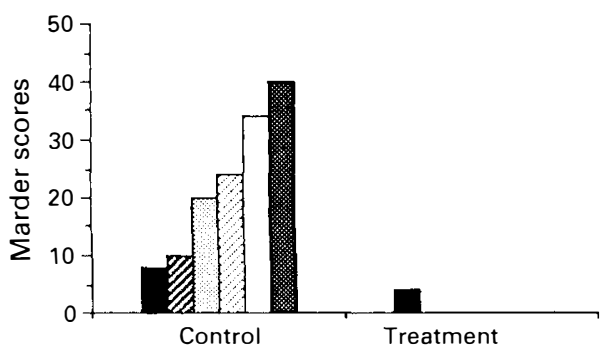

Figure 2 Scoring of DVTS using modified Marder scoring system

stasis, hypercoagulability, and intimal injury. Early DVT prophylaxis studies utilized subcutaneous heparin or warfarin to reduce hypercoagulability with the efficacy of treatment assessed clinically and not by diagnostic tests. ${ }^{14.15}$ A more rigorous placebo controlled study was completed using low dose heparin $(5,000$ units, SC, Q8 hrs) as the sole prophylactic agent. ${ }^{5}$ Effectiveness of therapy was confirmed by venography. The incidence of DVT was $49 \%$ in the placebo group and $52 \%$ in the heparin group, a nonsignificant difference. Green et al reported the use of adjusted dose subcutaneous heparin versus low dose heparin as a prophylaxis for DVT in acute spinal cord injured patients. ${ }^{16}$ This method utilized a standard dose of heparin which was adjusted on a sliding scale to maintain the PTT in the top normal range of the laboratory. This prophylaxis reduced the incidence from $31 \%$ in the low dose heparin group to $7 \%$ in the adjusted dose heparin group. Although this form of treatment was effective, it was associated with a high incidence of bleeding complications $(24 \%)$ for which prophylaxis had to be discontinued. These results raised the issue concerning the need for the evaluation of combination therapy or better dosing of single pharmacological agents for prophylaxis.

More recent studies regarding hypercoagulability in spinal cord injured patients have demonstrated an increase in platelet aggregation with a resultant increase in the risk of developing DVT. ${ }^{6}$ Using this finding Green et al compared EPC to EPC plus aspirin/dipyridamole as prophylaxis for DVT in acute SCI. ${ }^{8} 125$ I fibrinogen scanning with confirmatory venography for po- 
sitive scans was utilized to assess the efficacy of these prophylactic measures. DVT was reduced from $72 \%$ in the previously studied control group reported by the author to $35 \%$ in the EPC alone group and $25 \%$ in the EPC plus aspirin/dipyridamole group.

Another approach to combination prophylactic measures to reduce both stasis and hypercoagulability employed electrical stimulation (ES) of the calf plus low dose heparin $\left(5,000\right.$ units, SC, Q8 hrs). ${ }^{5}$ In this randomized, prospective, placebo controlled study all patients were evaluated for 4 weeks following acute SCI by surveillance 125 I fibrinogen scanning. Bilateral leg venography was performed upon completion of the protocol. All positive 125 I fibrinogen scans prior to completion of the study were confirmed by venography. This mode of prophylaxis reduced the incidence of DVT from $49 \%$ in the placebo group to $6.7 \%$ in the treated group.

In the previously reported studies by Merli et al and Rossi et al DVT was reported to occur most frequently during the first 2 weeks following spinal cord injury. ${ }^{5,6}$ Merli et al reported a $54 \%$ incidence between days 3 through 7 and 33\% days 8 through $14 .{ }^{17}$ Rossi et al noted a $62 \%$ incidence between day 6 to 8 and a $23 \%$ incidence day 9 through $14 .{ }^{6}$ Using this data it was decided that 14 days was an adequate study period.

The present study examines a different combination regimen for DVT/PE prophylaxis namely EPC, GES, and LDH. EPC has been shown to enhance venous blood flow in the lower extremities. This mechanical modality used alone as prophylaxis in neurosurgical, orthopedic, urological, and acute SCI patients has been shown to significantly decrease the incidence of DVT. ${ }^{18-20}$ Unlike electrical stimulation, which cannot be used in sensory intact candidates, EPC can be applied to all types of patients. GES were added to further enhance the reduction of stasis. This modality has been demonstrated to be effective in low risk surgery. ${ }^{21}$ The combination of the above modalities is designed to reduce stasis, one of the major arms of Virchow's Triad. Low dose heparin $(5,000 \mathrm{U}, \mathrm{SC}$, Q12 hrs) was chosen for its effect on reducing hypercoagulability, another arm of Virchow's Triad. The rationale for low dose heparin is the reduction of activated factor $\mathrm{Xa}$, resulting in decreased generation of thrombin and clot formation. ${ }^{22}$ This pharmacological agent has demonstrated effectiveness in multiple surgical studies and is far more effective in reducing DVT in the surgical population than aspirin or dipyridamole alone or in combination. ${ }^{23,24}$ The every 12 hours dosing schedule for low dose heparin was chosen instead of the every 8 hours for ease of administration since neither regimen has been shown to be more effective than the other. ${ }^{25}$ Low dose heparin alone has not been shown to decrease the incidence of DVT in the acute SCI population. ${ }^{5}$ The combination of low dose heparin to decrease hypercoagulability and EPC plus GES to decrease stasis in this study demonstrated a decrease in both the number and size of thrombi when compared to a historical control group. This decrease in size of thrombi may be an important finding since no other study in this population has demonstrated this change. The results indicate that this combination therapy may be a more effective approach to prophylaxis in the spinal cord injury population. Because this study has such a small sample size and utilized an historical control, a proper prospective, randomized trial will be necessary to confirm the efficacy of combination therapy in acute spinal cord injury.

\section{Acknowledgements}

This study was supported in part by the Regional Spinal Cord Injury Center of the Delaware Valley Model SCI Systems grant G008535135. We would like to thank Hyman Menduke PhD, Professor of Pharmacology (Biostatistics), Thomas Jefferson University, for his assistance in preparing statistical analysis.

\section{References}

1 Acute Traumatic Spinal Cord Injury Surveillance-United States 1987 (1988) Morbidity and Mortality Weekly Report 37: 285-286. 
2 Frankel H, Hancock D, Hyslop G, et al (1969-70) Value of postural reduction in initial management of closed injuries of spine with paraplegia and tetraplegia: part I. Paraplegia 7: 179-192.

3 Tribe CR (1963) Causes of death in early and late stages of paraplegia. Paraplegia 1: 19-47.

4 Stover SL, Fine DR, DeVivo MJ, Weitz H, Heuzes J, Park C (1983) University of Alabama in Birmingham Spinal Cord Injury Data Management Service. Quarterly Report for the period March 1, 1983 through June 30: $21-22$.

5 Merli GJ, Herbison GJ, Ditunno JF et al (1988) Deep vein thrombosis: prophylaxis in acute spinal cord injured patients. Arch Phys Med Rehabil 69: 661-664.

6 Rossi E, Green D, Rosen J, Spies S, Yao J (1980) Sequential changes in factor VIII and platelets preceding deep vein thrombosis in patients with spinal cord injury. Br J Haematol 45: 143-151.

7 Silver J (1974) The prophylactic use of anticoagulant therapy in the prevention of pulmonary emboli in one hundred consecutive spinal injured patients. Paraplegia 12: 188-196.

8 Green D, Rossi EC, Yao JST, Flinn W, Spies S (1982) Deep vein thrombosis in spinal cord injury: effect of prophylaxis with calf compression, aspirin, and dipyridamole. Paraplegia 20: 227-234.

9 Kakkar VV (1972) The diagnosis of deep vein thrombosis using the 125 I fibrinogen test. Arch Surg 104: 152-159.

10 Rabinov K, Paulin S (1972) Roentgen diagnosis of venous thrombosis in the leg. Arch Surg 104: $134-144$.

11 Marder VJ, Soulen RL, Atichartakarn V, Budzynski A, Parulekar S, Kim J (1977) Quantitative venography assessment of deep vein thrombosis in the evaluation of streptokinase and heparin therapy. J Lab Clin Med 89: $1018-1029$

12 Bross IDJ (1954) 'Is there an increased risk?' Federation Proceedings, September 1954. Federation of American Societies for Experimental Biology: 815-19.

13 Gruber UF, Thoni F (1985) Prevention of thrombo-embolism complications in paraplegia. Paraplegia 23: 124.

14 Hachen HJ (1974) Anticoagulant therapy in patients with spinal cord injury. Paraplegia 12: 176-187.

15 Watson N (1978-79) Venous thrombosis and pulmonary embolism in spinal cord injury. Paraplegia 3: 209-213.

16 Green D, Lee M, Ito V, Cohn T, Press J, Filbrandt P (1988) Fixed vs adjusted dose heparin in the prophylaxis of thromboembolism in spinal cord injury. JAMA 260: 1255-1258.

17 Merli G, Vollmer J, Heise R, Crabbe S, Weitz H, Sciana A (1990) Natural history of deep vein thrombosis in acute spinal cord injury. J Am Paraplegia Soc 13 (1): 53.

18 Black P, Crowell R, Abbott W (1986) External pneumatic calf compression reduces deep vein thrombosis in patients with ruptured intracranial aneurysm. Neurosurgery 18: 25-27.

19 Hartman JT, Pugh JL, Smith RD, Robertson W, Yost R, Janssen H (1982) Cylic sequential compression of the lower limbs in prevention of deep vein thrombosis JBJS (Am) 64: 1059.

20 Coe N, Collins R, Klein L, Bettmann M, Skillman J, Shapiro R (1978) Prevention of deep vein thrombosis in urologic patients: a controlled, randomized trial of low-dose heparin and external pneumatic compression boots. Surgery 83: 230-234.

21 Allan A, Williams JT, Bolton JP, Le Quesne J (1983) The use of graduated compression stockings in the prevention of postoperative deep vein thrombosis. Br J Surg 70: 172-174.

22 Wessler S (1975) Biochemical rational for low-dose heparin in deep venous thrombosis. In: Fratantoni J, Wessler S, editors. Prophylactic Therapy of Deep Vein Thrombosis and Pulmonary Embolism. DHEW. Pub. No (NIH) 76-866. Washington, D.C., GPO: 175-182.

23 An International Multi-Center Study (1975) Prevention of fatal postoperative pulmonary embolism by low doses of heparin. Lancet 2: 45-51.

24 Groote-Schuur Hospital Thromboembolism Study Group (1979) Failure of low dose heparin to prevent significant thromboembolism complications in high risk surgical patients. Interim report of a prospective trial. $B r$ Med J 1: 1447-1450.

25 Claggett G, Reisch J (1988) Prevention of venous thromboembolism in general surgical patients. Ann Surg 208: 227-240. 